Document downloaded from:

http://hdl.handle.net/10251/72768

This paper must be cited as:

López, JJ.; Salvador Rubio, FJ.; De La Garza De Leon, O.; Arregle, JJP. (2012). A comprehensive study on the effect of cavitation on injection velocity in diesel nozzles. Energy Conversion and Management. 64:415-423. doi:10.1016/j.enconman.2012.03.032.

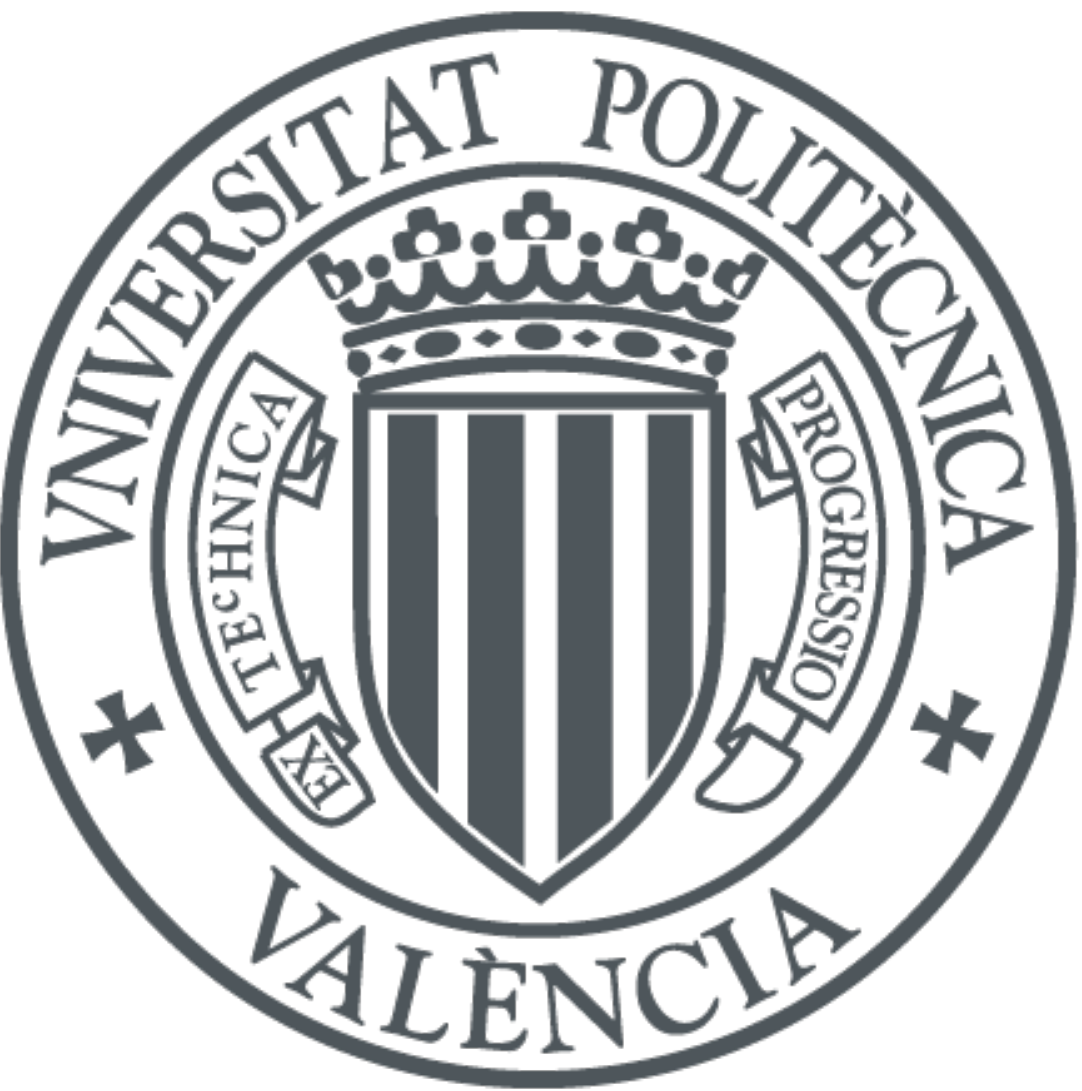

The final publication is available at

http://dx.doi.org/dx.doi.org/10.1016/j.enconman.2012.03.032

Copyright Elsevier

Additional Information 


\title{
A comprehensive study on the effect of cavitation on injection velocity in diesel nozzles
}

\author{
J. Javier López ${ }^{\mathrm{a}, *}$, F. Javier Salvador ${ }^{\mathrm{a}}$, Oscar A. de la Garza ${ }^{\mathrm{a}}$, Jean Arrègle ${ }^{\mathrm{b}}$ \\ ${ }^{a}$ CMT-Motores Térmicos \\ ${ }^{b}$ Departamento de Máquinas y Motores Térmicos. \\ Universitat Politècnica de València \\ Camino de Vera, s/n. 46022 Valencia, SPAIN
}

\begin{abstract}
Results when testing cavitating injection nozzles show a strong reduction in mass flow rate when cavitation appears (the flow is choked), while the momentum flux is reduced to a lesser extent, resulting in an increase in effective injection velocity. So as to better understand the origin of this increase in effective injection velocity, the basic equations for mass and momentum conservation were applied to an injection nozzle in simplified conditions.

The study demonstrated that the increase in injection velocity provoked by cavitation is not a direct effect of the latter, but an indirect effect. In fact, the vapor appearance inside the injection hole produces a decrease in the viscosity of the fluid near the wall. This leads to lower momentum flux losses and to a change in the velocity profile, transforming it into a more "top hat" profile type. This change in the profile shape allows explaining why the momentum flux reduction is not so important compared to that of the mass flow rate, thus explaining why the effective injection velocity increases.
\end{abstract}

\footnotetext{
*Corresponding author

Tel: +34963 879 232. Fax: +34963877 659. E-mail: jolosan3@mot.upv.es
} 
Keywords: Nozzle cavitation, velocity profile, injection velocity

\section{Introduction}

Diesel engine designers are continuously trying to improve the combustion process to satisfy both the customer demands and the legislation requirements concerning pollutant emissions. Nowadays several different paths are followed to achieve these goals: the redefinition of the combustion process, the use of multiple injection strategies, the use of alternative fuels, etc. Just a few examples of these paths will be reviewed in the following lines. As it has been just said, one of the paths is the redefinition of the combustion process. Gan et al. [1], among other authors, investigate the HCCI (Homogeneous Charge Compression Ignition) combustion mode, reporting that it is an appropriate alternative to reduce both NOx and soot emissions. Torregrosa et al. [2] investigated the potential of the PCCI (Premixed Charge Compression Ignition) combustion mode, concluding that it is an attractive strategy to fulfil pollutant emissions requirements although the engine noise is strongly deteriorated. Another possible path is the use of multiple injection strategies. Thurnheer et al. [3], for instance, compared different injection strategies (including pilot, main and post-injection) in a heavy-duty diesel engine. They found the advantages and disadvantages, from the point of view of efficiency and pollutant emissions, of each strategy as a function of the timing and size of the pilot and/or post-injection. Arrègle et al. [4] showed the strong potential of the post-injection to reduce soot emissions, and they performed a thorough study trying to understand the reasons for this potential. Suh et al. [5] performed an investigation of multiple injection 
strategies in combination with an oxygenated fuel, showing that a simultaneous reduction of soot and NOx emissions could be achieved. Finally, the later mentioned path is the use of alternative fuels. Ozsezen and Canakci [6] and Qi et al. [7], reported significant benefits of the use of biodiesel fuel (instead of standard diesel fuel) concerning $\mathrm{CO}$ and soot emissions, although NOx emissions were slightly higher.

Among the paths taken nowadays to fulfil the customer demands and the legislation requirements, the design of the injection nozzle in a diesel engine is an important factor to improve the combustion process and, above all, to reduce pollutant emissions, as demonstrated by, for example, Karra and Kong [8] and Som et al. [9], who showed that the nozzle geometry have a significant impact on soot emissions. The main reason for this effect is that the nozzle geometry has an influence on the internal flow and on the spray characteristics, particularly on the atomization [10, 11] and mixing [12, 13] processes. This is the main reason why there are lots of studies analyzing the effect of nozzle geometry on its internal and external flow $[14,15]$. One of the aspects the nozzle geometry has an influence on is the appearance or not of the cavitation phenomenon. It is well-known that, even if this phenomenon has a clear negative effect on the permeability, it also has a positive effect on the atomization (Soteriou et al.[16], as well as Suh and Lee [17], reported an improvement of the atomization process caused by cavitation) and the mixing process (Desantes et al. [18], for instance, showed that cavitation provokes a significant increase in the initial spray angle, which translates into a better mixing process). For this reason it has been widely studied.

The effect of cavitation on the flow in an injection nozzle usually has been 
analyzed in terms of what happens with the mass flow rate. Concerning this parameter, it can be stated that it is significantly reduced by cavitation. In fact, the mass flow rate reaches choking conditions when the injection pressure is kept constant and the back-pressure is reduced below a certain limit, as shown in Fig. 1 using some results already published [19]. In those experiments 5 different injection pressure levels were used, and for each of them the back-pressure was modified. The experiments were conducted with standard diesel fuel, with a density of $820 \mathrm{~kg} / \mathrm{m}^{3}$ and a dynamic viscosity of $2.19 \cdot 10^{-3} \mathrm{~kg} / \mathrm{m} \cdot \mathrm{s}$. Figure 1 shows, on the one hand, results corresponding to a conical nozzle (i.e. without cavitation) and a cylindrical nozzle (i.e. with cavitation under some working conditions), both having a similar Bosch Flow Number ${ }^{1}$. The main characteristics of both nozzles are shown in Table 1. The previous Figure also shows, on the other hand, the evolution of the mass flow rate (to the left) and the discharge coefficient (to the right) of both nozzles. The discharge coefficient is defined as follows:

$$
C_{d}=\frac{\dot{m}}{\rho_{L} \cdot A_{o} \cdot u_{\text {berno }}}
$$

where $\dot{m}$ is the mass flow rate, $\rho_{L}$ is the density of the liquid fuel, $A_{o}$ is the geometric cross-sectional area of the orifice, and $u_{\text {berno }}$ is the Bernoulli's velocity. In both curves it can be clearly observed the strong effect of cav-

\footnotetext{
${ }^{1}$ This parameter represents the volume of fuel injected during 30 seconds using an injection pressure and a back-pressure of 10 and $0.1 M P a$, respectively. It is worthy to underline that a lot of cavitation exists in the cylindrical nozzle under these conditions, and consequently the permeability of this nozzle will be significantly higher than the other when there is no cavitation.
} 

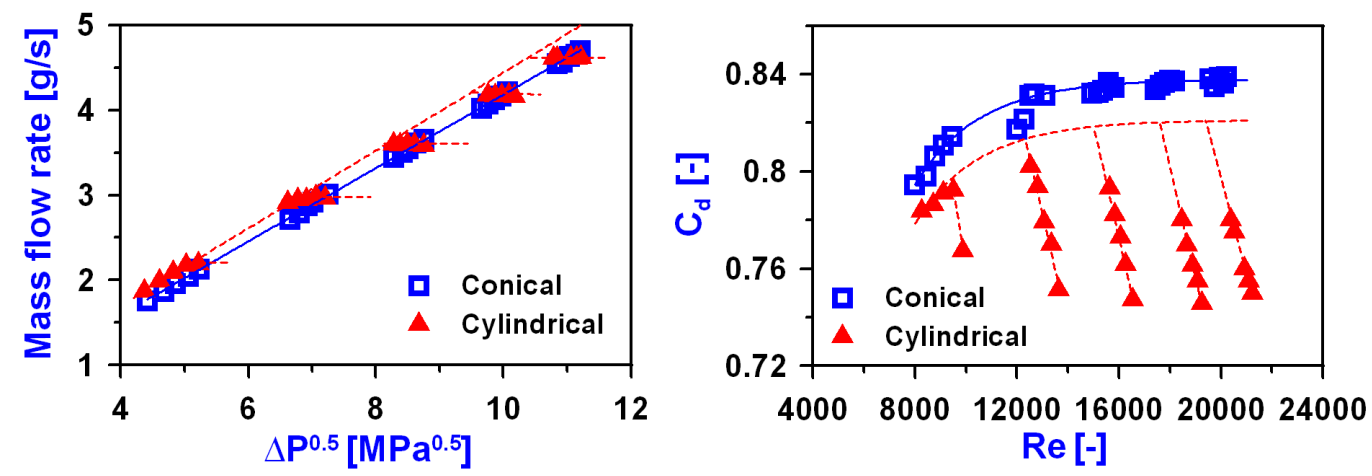

Figure 1: Results for a cylindrical (potentially cavitating) and a conical (non-cavitating) nozzle with identical Bosch Flow Number. Left.- Mass flow rate as a function of $\Delta p^{0.5}$. Right.- Discharge coefficient $\left(C_{d}\right)$ as a function of $R e$.

\begin{tabular}{|l|c|c|}
\hline \hline Nozzle & $d_{o}[\mu \mathrm{m}]$ & Bosch Flow Number [cc/30 s.] \\
\hline Cylindrical & 131 & 97.2 \\
\hline Conical & 125 & 97.6 \\
\hline \hline
\end{tabular}

Table 1: Main geometrical characteristics of the two-orifice nozzles used in [19]. $d_{o}$ is the outlet diameter. 
itation on this aspect (cylindrical nozzle). This is a very common result in the literature (e.g. Desantes et al. [18] and Payri et al. [20], among many other authors, have obtained the same behaviour), and its justification has been known for more than 30 years [21]. In that work, Nurick states that when the vapor pressure is achieved at the minimum area orifice section, this pressure level can not be reduced anymore even if the pressure at the discharge enclosure is further reduced. This fact, similarly as what happens in a convergent-divergent nozzle where the sonic conditions at the "throat" are achieved, lead to blocking the mass flow rate.

In Fig. 1, to the right, it can be clearly observed that the flow through a nozzle (quantified by the $C_{d}$ ) depends both on the $R e$ and on the cavitation level, usually quantified by the Nurick Cavitation Number, $K$, defined as follows:

$$
K=\frac{p_{i n j}-p_{v a p}}{p_{i n j}-p_{\text {back }}}
$$

where $p_{i n j}$ is the injection pressure, $p_{\text {back }}$ is the back-pressure, and $p_{v a p}$ is the fuel vapor pressure. If the experimental procedure commonly used for the study of cavitation (to keep a constant injection pressure modifying only the back-pressure) is analyzed in a critical way, it can be seen that both $R e$ and $K$ are changed at the same time. A better way to proceed to study cavitation is to change only $K$ while keeping a constant $R e$. This can be done working at a constant $\Delta p\left(p_{i n j}-p_{\text {back }}\right)$, i.e. modifying $p_{i n j}$ and $p_{\text {back }}$ at the same time.

When working with this methodology with different nozzles and injector holders, it is observed that sometimes the effect of $R e$ on the flow is negligible whereas some other times it is very important. From the authors experience, 
usually single-orifice nozzles show a small dependence of the $R e$ on the flow, whereas multi-orifice nozzles show a significant dependence. The reason for this behavior, however, is not yet understood. The example that will be used later to more deeply show the effect of cavitation on the flow, corresponds to two nozzles (a conical and a cylindrical nozzle) with a single orifice in which the $R e$ is not important at all. This way the attention will be focused on the effect of $K$, i.e. on cavitation.

Some measurements of the mass flow rate and momentum flux have been performed with these two nozzles, as had been already done in many previous studies using the traditional methodology [19, 22]. When analyzing the momentum flux, a momentum coefficient is defined (in a similar way to the discharge coefficient for the mass flow rate) as follows:

$$
C_{M}=\frac{\dot{M}}{\rho_{L} \cdot A_{o} \cdot u_{\text {berno }}^{2}}
$$

where $\dot{M}$ is the momentum flux. From these two coefficients $\left(C_{d}\right.$ and $\left.C_{M}\right)$, the velocity coefficient relating the effective velocity (i.e. the actual injection velocity) and the Bernoulli's velocity can be defined:

$$
u_{e f f}=\frac{\dot{M}}{\dot{m}}=C_{v} \cdot u_{\text {berno }} \quad \Rightarrow \quad C_{v}=\frac{C_{M}}{C_{d}}
$$

In Fig. 2 the value for these three coefficients is shown only for the cylindrical nozzle (the case where cavitation can appear). The different data have been presented in the following way: each coefficient has been divided by its corresponding value when there is no cavitation, in such a way that they have a value of 1 in the non-cavitating region. From this Figure, it can be observed that: 


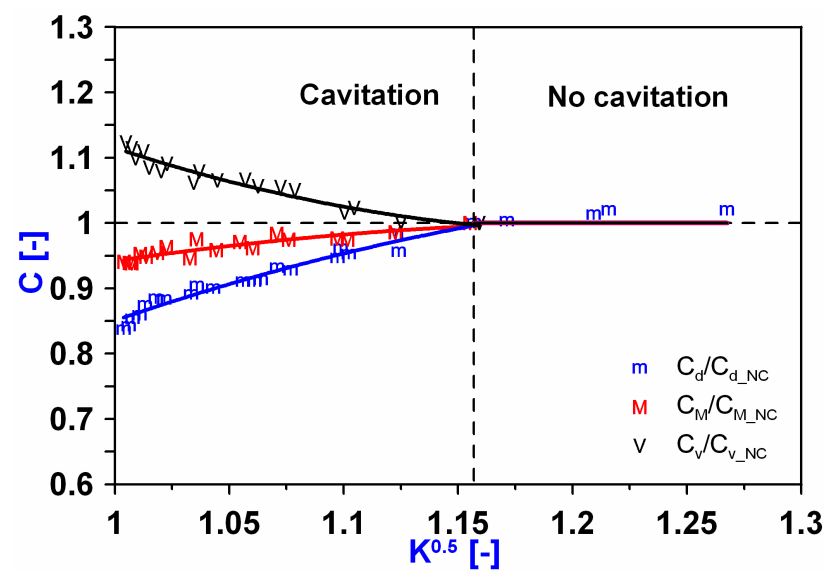

Figure 2: Evolution of $C_{d}, C_{M}$ and $C_{v}$ for a cylindrical nozzle (i.e. a potentially cavitating nozzle). The values have been normalized by the corresponding non-cavitating value.

- The mass flow rate, represented by $C_{d}$, is strongly reduced as the cavitation intensity increases (i.e. as $K$ approaches the value 1 ).

- The momentum flux, represented by $C_{M}$, is also reduced as the cavitation intensity increases, but to a lesser extent compared to the mass flow rate.

- As a consequence of the two previous observations, the effective injection velocity, represented by $C_{v}$, increases as the cavitation intensity increases.

As a conclusion, cavitation provokes an increase in injection velocity. The objective of this work is to analyze the causes for this increase, trying to find out if it is a direct consequence of cavitation or if it is an indirect consequence, i.e. that cavitation produces some changes in the fluid that would lead, as a secondary consequence, to an increase in velocity. 
To perform this study a very simplified case (in order to allow for an analytical solution) will be analyzed to find out which are the "expected" theoretical effects (i.e. direct effects) of cavitation on flow velocity. This result will be then compared to some CFD results and some experimental results to try to better understand the mechanisms of the effect of cavitation on the flow velocity.

\section{Theoretical analysis of the direct effects of cavitation}

Because of the enormous difficulty when trying to analytically solve a cavitating flow in a nozzle, the theoretical analysis that will be performed here is going to be done in a very simplified case. Further in this study the validity and scope of the results obtained here will be discussed. This analysis is closely related to the one performed by Nurick [21] and the one performed later by Schmidt and Corradini [23]. The main hypotheses are the following:

- An orifice configuration as the one shown in Fig. 3, to the left, is assumed. It is worthy to underline that the orifice (geometrically cylindrical), because of the fluid dynamics, presents a narrowing provoked by a recirculation zone that makes it similar to a convergent-divergent nozzle (independently of the existence or not of cavitation). Three interesting cross-sections have been marked in the Figure: section 1, at the inlet, where the velocity is negligible; section $c$, at the narrowing, where the cross-sectional area has the minimum value; and section 2 , at the exit of the orifice. 

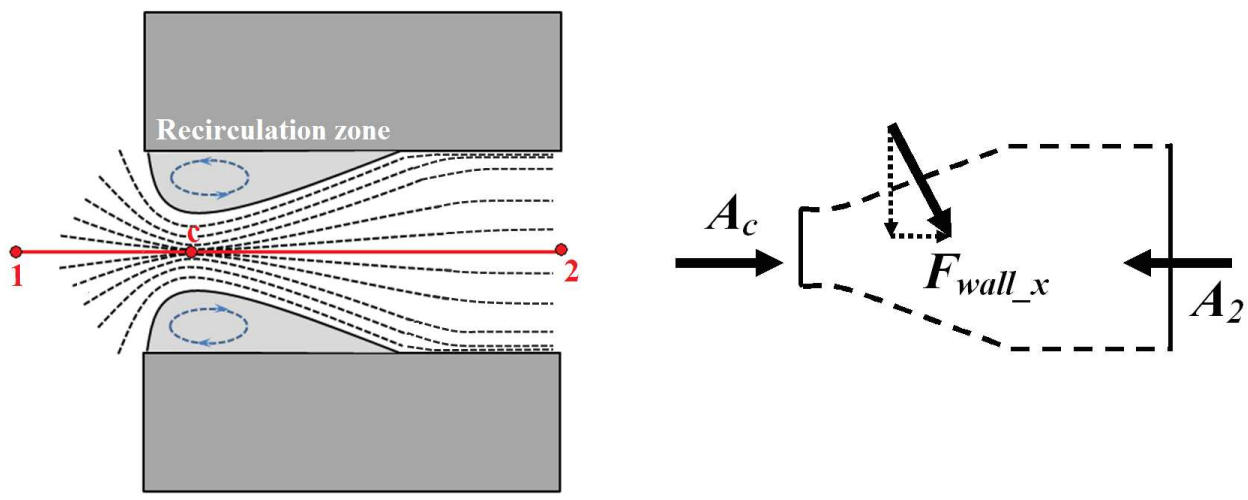

Figure 3: Left.- Schematics of the orifice configuration. Right.- Control volume between sections $c$ and 2 .

- The fluid and flow properties are assumed to be uniform at each section. This means that $C_{d}$ is 1 when no cavitation exists (remember that an ideal case is considered).

- Friction between the fluid and the wall is neglected.

- The cavitating case to be analyzed will be the one where $p_{2}=p_{\text {vap }}$. As it will be shown later, this is the only case where an easy analytical solution can be obtained. The consequences of this particular selection will be discussed later.

The only equations to be used are the continuity equation, the momentum theorem and the energy equation. The latter, if particularized to an incompressible fluid translates into the Bernoulli's theorem (obviously, this equation can only be used in the regions where a single-phase flow exists).

To perform the theoretical analysis, a cavitating case will be compared to a non-cavitating one. The particular cavitating case, as mentioned above, is 
the one where the pressure at the orifice outlet is precisely the vapor pressure (in this case, the pressure between the throat and the exit is uniform and equal to $\left.p_{\text {vap }}\right)$. The non-cavitating case is a case with the same $\Delta p$ between the inlet and the outlet, but with a $p_{c}$ at the throat higher than the vapor pressure (in order to avoid cavitation).

Starting by the non-cavitating case, as a single-phase flow exists in all the intermediate sections, the mass flow rate and the momentum flux at the outlet section can be directly calculated by using the basic equations for incompressible flow:

$$
\dot{m}_{w / o_{-} c a v}=\rho_{L} \cdot A_{2} \cdot u_{2 \_w / o \_c a v}
$$

where $u_{2 \_w / o \_c a v}$ can be obtained from Bernoulli's equation:

$$
\begin{aligned}
& u_{2 \_w / o \_c a v}=\left(\frac{2 \cdot\left(p_{1}-p_{2}\right)}{\rho_{L}}\right)^{0.5} \\
& \dot{M}_{w / o_{-} c a v}=\rho_{L} \cdot A_{2} \cdot u_{2 \_w / o \_c a v}^{2}
\end{aligned}
$$

For the cavitating case, the mass flow rate can be written in the following way:

$$
\dot{m}_{w_{\_} c a v}=\rho_{L} \cdot A_{c} \cdot u_{c \_w \_c a v}=\rho_{2} \cdot A_{2} \cdot u_{2 \_w \_c a v}
$$

where the value of $u_{c \_w \_c a v}$ can be obtained from Bernoulli's equation (because between section 1 and $c$ there is a single-phase flow):

$$
u_{c \_w \_c a v}=\left(\frac{2 \cdot\left(p_{1}-p_{c}\right)}{\rho_{L}}\right)^{0.5}
$$


As $p_{c}=p_{2}=p_{v a p}$, from Eqs. (6) and (9) it can be deduced that $u_{c_{-} w_{-} c a v}=$ $u_{2 \_w / o \_c a v}$ (because the pressure difference $p_{1}-p_{2}$ is the same in both cases).

For the momentum flux (in the axial direction, i.e. the $x$-direction) it can be written:

$$
\dot{M}_{w \_c a v}=\rho_{2} \cdot A_{2} \cdot u_{2 \_ \text {w_cav }}^{2}
$$

Now this momentum flux at the exit will be related to the one at the throat, because its value can be known at this latter cross-section. If the momentum theorem is applied between section $c$ and 2 (the control volume considered is shown in Fig. 3 to the right), the following expression is obtained (only the pressure forces are taken into account ${ }^{2}$, because friction was considered to be negligible):

$$
p_{c} \cdot A_{c}-p_{2} \cdot A_{2}+F_{\text {wall_x }}=\Delta \dot{M}
$$

$F_{\text {wall_x }}$ can be obtained as the integration of the pressure forces. The expression can be easily integrated because pressure is constant in all the considered control volume (this is the main reason to select this particular case for the analysis):

$$
F_{\text {wall_x }}=\int_{c}^{2} p \cdot d A=p_{\text {vap }} \cdot\left(A_{2}-A_{c}\right)
$$

Substituting Eq. (12) into Eq. (11), taking into account that $p_{c}=p_{2}=$ $p_{\text {vap }}$, it is obtained:

\footnotetext{
${ }^{2}$ It is worthy to note that the term $F_{\text {wall }} x$ refers to the axial component of the force performed by the wall to the fluid due to the pressure of the latter.
} 


$$
p_{c} \cdot A_{c}-p_{2} \cdot A_{2}+p_{2} \cdot A_{2}-p_{c} \cdot A_{c}=0=\Delta \dot{M}
$$

thus deducing that the momentum flux is the same in sections $c$ and 2. Then, Eq. (10) can be written as follows:

$$
\dot{M}_{w_{\_} c a v}=\rho_{2} \cdot A_{2} \cdot u_{2 \_w \_c a v}^{2}=\rho_{L} \cdot A_{c} \cdot u_{c \_w \_c a v}^{2}
$$

Taking into account these results, the effect of cavitation on the mass flow rate and the momentum flux can be found. Concerning the mass flow rate, if Eq. (5) is divided by Eq. (8), it is obtained:

$$
\frac{\dot{m}_{w / o \_c a v}}{\dot{m}_{w \_c a v}}=\frac{\rho_{L} \cdot A_{2} \cdot u_{2 \_w / o \_c a v}}{\rho_{L} \cdot A_{c} \cdot u_{c_{-} w_{-} c a v}}=\frac{A_{2}}{A_{c}}
$$

And concerning the momentum flux, if Eq. (7) is divided by Eq. (14), it is found:

$$
\frac{\dot{M}_{w / o \_c a v}}{\dot{M}_{w \_c a v}}=\frac{\rho_{L} \cdot A_{2} \cdot u_{2 \_w / o \_c a v}^{2}}{\rho_{L} \cdot A_{c} \cdot u_{c \_ \text {w cav }}^{2}}=\frac{A_{2}}{A_{c}}
$$

As can be seen, the result is the same as for the mass flow rate. Besides, for the cavitating case, as both the mass flow rate and the momentum flux are the same in sections $c$ and 2, it can be deduced that velocity is also the same, and it can be written that $u_{2 \_w_{-} c a v}=u_{c_{-} w_{-} c a v}=u_{2 \_w / o_{-} c a v}$.

Consequently, based on the particular case analyzed here, the effect of cavitation on $\dot{m}$ and $\dot{M}$ is theoretically the same, and thus the exit velocity would not be altered. This means that the cavitation process, which translates mainly in a reduction in density due to the appearance of a two-phase flow, does not directly produce an increase in flow velocity. Even if this 
statement is only valid for a very particular case as the one analyzed here (remember that this case was selected because to analytically solve the problem in any other pressure conditions is extremely more complicated), there is a fact that can help to generalize the result. The case analyzed corresponds to the case where $p_{2}=p_{v a p}$, and in these conditions the cavitation intensity is huge. If with this level of cavitation, much higher than the one that can be found in a diesel nozzle (because $p_{2}$-the in-cylinder pressure- is always much higher than $\left.p_{\text {vap }}\right)$, there is no increase in velocity, it can be thought that the velocity will not increase for any other lower cavitation level. As a conclusion, even if this theoretical result has been obtained for a very particular case, it seems that the result (that cavitation does not directly provoke an increase in the flow velocity) can be considered as a general result.

Once this point has been reached, if cavitation doesn't have a direct effect on the increase in effective injection velocity, it is important to wonder which parameter is affected by cavitation that may be responsible for this increase in velocity. The hypothesis to be considered here is that cavitation produces a decrease in fluid viscosity, the effect of which can be observed from two complementary points of view:

- On the one hand, wall friction is reduced where cavitation appears (i.e. between sections $c$ and 2). In the theoretical development just shown above, wall friction has been neglected so as to be able to obtain an analytical solution for the analyzed case. If this friction were considered, it would be found that momentum flux between sections $c$ and 2 would decrease to a lesser extent in the cavitating case compared to the non-cavitating case, which would explain why the momentum flux 
reduction is actually smaller compared to what ideally (i.e. without friction) would be expected.

- On the other hand, the decrease in viscosity leads to an increase in $R e$, thus increasing the turbulent character of the flow. This would lead to a transformation of the velocity profile towards a more "top hat" shape (velocity will keep a higher value near the wall) [24]. This change in the velocity profile, which is in fact related to what has been explained in the previous item, is what can explain why the decrease in mass flow rate and in momentum flux is not the same.

The effect of the fluid viscosity on the velocity profile (i.e. the latter point of view) will be analyzed in next section.

\section{Analysis of the effect of viscosity (without cavitation) on the velocity profile}

It has just been said that the viscosity drop caused by cavitation would lead to a more "top hat" velocity profile. This change in the velocity profile shape could possibly explain that the effect of cavitation on $\dot{m}$ (which is proportional to $\int u$ ) and on $\dot{M}$ (proportional to $\int u^{2}$ ) would be different.

In order to validate this hypothesis, some simulations with the CFD code OpenFOAM $[25,26]$ will be used. This code was previously validated under diesel conditions in [27], in both cavitating and non-cavitating conditions. The main characteristics and parameters for the simulations are summarized in Table 2. The effect of the fluid viscosity (of all the fluid, i.e. not considering a two-phase flow) on the flow through a convergent (so as to avoid cavitation 
and, consequently, a two-phase flow), single-orifice nozzle was studied. The orifice diameter was $112 \mu \mathrm{m}$ and its length was $1 \mathrm{~mm}$. The turbulence model was a $k-\varepsilon$ RNG model, with standard values for the different parameters. Because of the axisymmetry of the geometry, a 2D-axisymmetric mesh of 32,000 cells was defined, which is shown in Fig. 4. The grid-independence of the results was checked in [28], where the same mesh was used. It is worthy to note that the calculation results were compared to some experimental data available for this nozzle and the agreement was good. For the reference case, with the higher viscosity level (see more details below), the near-wall $y+$ (the dimensionless wall distance, defined e.g. in [29]) is 1.6, which indicates a more than acceptable accuracy of the mesh to analyse the velocity profiles [29]. For the calculations used here, an injection pressure of $71 \mathrm{MPa}$ and a backpressure of $1 \mathrm{MPa}$ were considered, and the velocity profiles at the outlet section for three different viscosity values were compared:

- Liq_visc $=3.67 \cdot 10^{-3} \mathrm{~kg} /(\mathrm{m} \cdot \mathrm{s})$ (corresponding to liquid diesel fuel), which is considered to be the reference case,

- Liq_visc $\times 0.2=7.34 \cdot 10^{-4} \mathrm{~kg} /(\mathrm{m} \cdot \mathrm{s})(80 \%$ smaller than that of the liquid diesel fuel, which would correspond to the viscosity of a blend of liquid and vapor diesel fuel with a vapor mass fraction of 0.8 , as explained later), and

- Liq_visc $\times 0.04=1.829 \cdot 10^{-4} \mathrm{~kg} /(\mathrm{m} \cdot \mathrm{s})(95 \%$ smaller than that of the liquid diesel fuel, corresponding to the viscosity of a blend of liquid and vapor diesel fuel with a vapor mass fraction of 0.95 ).

For determining these viscosity levels, the method currently used in com- 


\begin{tabular}{|l|l|}
\hline \hline Calculation type & 2 D axisymmetric \\
\hline Number of cells & 32,000 \\
\hline Turbulence model & $k-\varepsilon$ RNG \\
\hline Flow type & Incompressible \\
\hline Orifice geometry & Conical (convergent) \\
\hline Outlet diameter & $112 \mu \mathrm{m}$ \\
\hline Orifice length & $1 \mathrm{~mm}$ \\
\hline Fluid density & $835 \mathrm{~kg} / \mathrm{m}^{3}$ \\
\hline Fluid viscosity & $3.67 \cdot 10^{-3} / 7.34 \cdot 10^{-4} / 1.829 \cdot 10^{-4} \mathrm{~kg} /(\mathrm{m} \cdot \mathrm{s})$ \\
\hline \hline
\end{tabular}

Table 2: Main characteristics and parameters of the simulations performed with OpenFOAM.

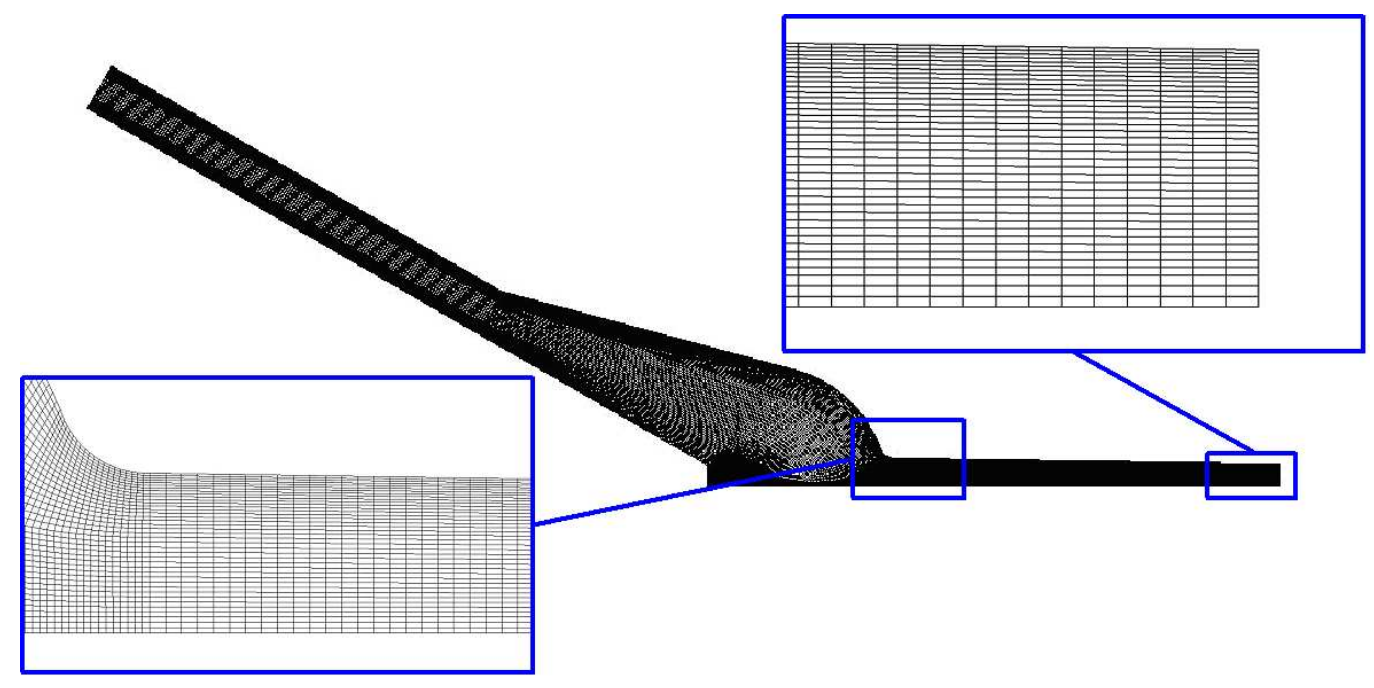

Figure 4: Details of the mesh used for CFD calculations with OpenFOAM. 
mercial CFD codes was used, i.e. assuming that the viscosity of a blend is the weighted average, as a function of the vapor mass fraction, of the liquid and vapor viscosity: $\mu_{m}=\mu_{L} \cdot\left(1-Y_{v a p}\right)+\mu_{v a p} \cdot Y_{v a p}$ (as in, e.g. [27]). The authors are aware of the unlikely hypothesis laying behind this method, but the validity of this common way for computing the viscosity of a liquid-vapor blend is out of the scope of this paper.

In Fig. 5, the profile of effective viscosity (defined as the addition of the molecular viscosity and the turbulent viscosity) at the outlet section as a function of the normalized radial coordinate $(\xi$, defined as the ratio between $r$, the radial coordinate, and $R$, the radius) is shown. On the one hand, it can be observed that the effective viscosity values approximately keep the proportion of the introduced laminar viscosities (i.e. the proportionality factors between the different effective viscosities are approximately the same as those for laminar viscosities), and thus changing the laminar viscosities was a successful method to obtain differences in the actual (i.e., effective) viscosity levels. On the other hand, the evolution of this parameter as a function of the radial coordinate shows a maximum near the wall. In this region the fluid velocity has the biggest change (maximum gradient), thus having a maximum turbulence intensity. This fact could justify the existence of a maximum in effective viscosity.

In Fig. 6, the velocity profiles for the three corresponding cases are shown. It can be clearly observed that viscosity affects very significantly the velocity profile and in the expected way: as the viscosity is reduced, the profile is more similar to a "top hat" profile. The reason for this behavior is that a reduction in viscosity leads to an increase in $R e$, thus leading to a more 


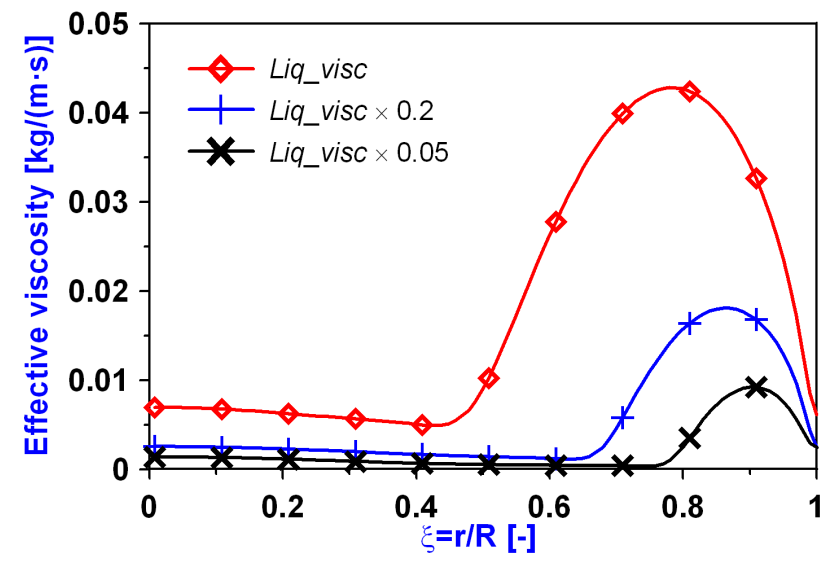

Figure 5: Effective viscosity profiles as a function of the normalized radial coordinate for the three viscosity levels simulated.

turbulent flow, which typically have "top hat" velocity profiles [24]. As a reference of the impact of this change in velocity profile, the $C_{d}$ for the three viscosity levels moved from 0.808 (this value, corresponding to the viscosity of standard diesel fuel, was validated with experimental data and the agreement was good) to 0.903 and 0.932 .

For these three obtained profiles, the effective velocity value, computed as $\dot{M} / \dot{m}$, was obtained. The results are shown in Table 3 (both absolute and relative -respect to the higher viscosity case- results are given). The obtained values are similar to the experimental results previously shown (Fig. 2). It can be stated, then, that the formulated hypothesis (that there is a change in the velocity profile caused by the variation in viscosity produced by cavitation) can suitably explain the experimental results: cavitation has a stronger effect on $\dot{m}$ than on $\dot{M}$, thus increasing the effective velocity at the nozzle exit. 


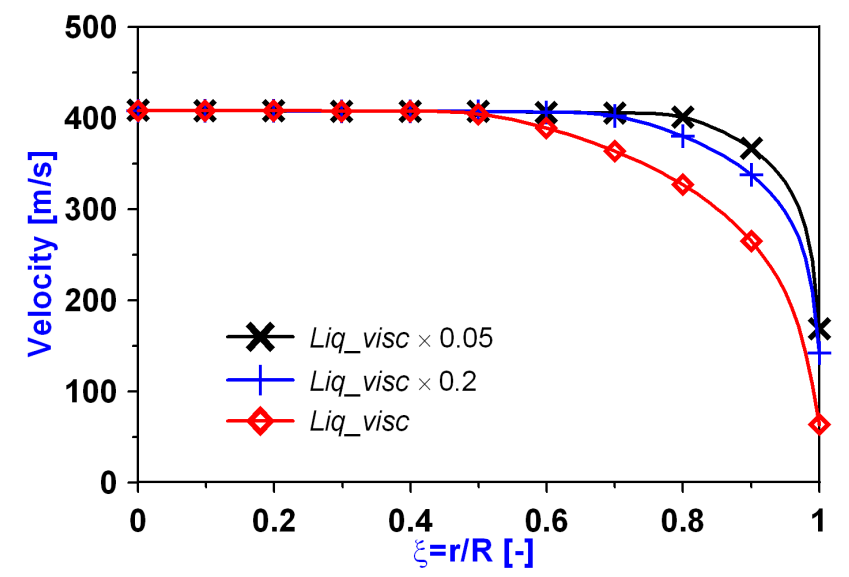

Figure 6: Velocity profiles as a function of the normalized radial coordinate for the three viscosity levels simulated.

\begin{tabular}{|l|c|c|c|}
\hline \hline Case & $u_{\text {eff }}=\dot{M} / \dot{m}[\mathrm{~m} / \mathrm{s}]$ & $u_{\text {eff }}$ ratio $[-]$ & $Y_{\text {vap }}[-]$ \\
\hline \hline Liq_visc & 354.0 & 1.000 & 0 \\
\hline Liq_visc $\times 0.2$ & 380.3 & 1.074 & 0.8 \\
\hline Liq_visc $\times 0.05$ & 389.8 & 1.101 & 0.95 \\
\hline \hline
\end{tabular}

Table 3: Effective velocity and its proportional change respect to the first case, and vapor mass fraction for the three viscosity levels. 
When viewing these results, a possible criticism that can be launched is that the viscosity variation that leads to a significant alteration of the velocity profile that, in turn, produces an increase in injection velocity similar to the one obtained experimentally is excessively high. Here it is important to point out that in these simulations the viscosity drop (which in the real case is associated to the appearance of vapor) is introduced uniformly in the whole section, whereas in the real case the vapor generated by cavitation is mostly concentrated near the walls [30, 9]. Consequently, for global vapor mass fractions relatively small and coherent with experimental results, the local vapor mass fraction near the wall and the corresponding viscosity drop are much more important. Besides, the viscosity near the wall is the one responsible for the change in velocity profile (because this one changes mostly in the region near the wall). This is what will be validated in the next section.

\section{Analysis of the effect of local variations in vapor mass fraction and viscosity on the velocity profile}

Finally, trying to enlarge the validity of the results obtained up to now, some simulations with the CFD code Star-CD were performed, using a cavitation model based on a linear representation of the Rayleigh-Plesset equation $[31,32]$. The main characteristics for these simulations are given in Table 4 . It is worthy to note that, on the one hand, now the nozzle has a cylindrical shape to allow cavitation appearance. On the other hand, the turbulence model (the standard $k-\varepsilon$ turbulence model) is different compared to the one used in the previous section (with OpenFOAM, a $k-\varepsilon$ RNG turbulence model was used). This other model was considered here because it is 
simpler, allowing slightly faster calculations, which is a critical issue in 3D calculations, and because the results are quite independent of the turbulence model, as demonstrated in [33]. The mesh used is a 3D mesh with around 57,000 cells, and it is shown in Fig. 7. As in the previous simulations, the grid-independence of the results was checked in [34], where the same mesh was used, and the results were compared with some experimental results available for this nozzle, the agreement of which was good. Three sets of simulations were performed, where the pressure drop across the orifice was kept constant, thus maintaining the Reynolds number, in each set. To achieve this, the injection pressure and the back-pressure were modified at the same time. The simulated cases were (they are identified by the couple injection pressure-back-pressure):

- Set 1: 31 - $1 M P a, 36-6 M P a, 40-10 M P a$ and 48 - $18 M P a$.

- Set 2: 71 - $1 M P a, 76-6 M P a, 80-10 M P a$ and 88-18 MPa.

- Set 3: 141 - $1 M P a, 146$ - $6 M P a, 150-10 M P a$ and 158 - $18 M P a$.

For the case $71-1 M P a$, which is equivalent to the reference case of the previous section, the near-wall $y+$ is 4.8 (i.e., three times higher compared to that other case). The main reason for this lower mesh resolution is simply because now the mesh is $3 \mathrm{D}$, whereas it was $2 \mathrm{D}$ in the previous section. Despite this lower accuracy in the mesh, it can be considered acceptable enough to analyse the velocity profiles [29].

In Fig. 8, the velocity profiles at the orifice outlet are shown for two sample cases: one with an injection pressure of $80 \mathrm{MPa}$ and a back-pressure of $10 \mathrm{MPa}$ (without cavitation) and the other with an injection pressure 


\begin{tabular}{|l|l|}
\hline \hline Calculation type & $3 \mathrm{D}$ \\
\hline Number of cells & $\sim 57,000$ \\
\hline Turbulence model & Standard $\mathrm{k}-\varepsilon$ \\
\hline Cavitation model & Linear representation of Rayleigh-Plesset equation \\
\hline Orifice geometry & Cylindrical \\
\hline Orifice diameter & $163 \mu \mathrm{m}$ \\
\hline Orifice length & $1 \mathrm{~mm}$ \\
\hline Fluid density & $835 \mathrm{~kg} / \mathrm{m}^{3}$ \\
\hline Fluid viscosity & $3.67 \cdot 10^{-3} \mathrm{~kg} /(\mathrm{m} \cdot \mathrm{s})$ (Liquid) \\
\hline \hline
\end{tabular}

Table 4: Main characteristics and parameters of the simulations performed with Star-CD.

\begin{tabular}{|l|c|c|c|}
\hline \hline Case & $u_{\text {eff }}=\dot{M} / \dot{m}[\mathrm{~m} / \mathrm{s}]$ & $u_{\text {eff ratio }[-]}$ & $C_{d}[-]$ \\
\hline \hline $80-10 \mathrm{MPa}$ & 367.23 & 1.0 & 0.851 \\
\hline $71-1 \mathrm{MPa}$ & 384.07 & 1.046 & 0.807 \\
\hline \hline
\end{tabular}

Table 5: Effective velocity and $C_{d}$ for the two cases simulated with Star-CD.

of $71 \mathrm{MPa}$ and a back-pressure of $1 \mathrm{MPa}$ (with cavitation). It can be observed that the trend is exactly the same as the one previously observed and discussed, reinforcing the result about the change in the velocity profile caused by cavitation. The numerical values of the effective velocity (both the absolute -computed as $\dot{M} / \dot{m}$ - and the relative values -respect to the noncavitating case- are shown) and the $C_{d}$ for both cases are given in Table 5 .

In Figs. 9 and 10, respectively, the radial evolution of the vapor mass fraction inside the nozzle and the effective viscosity for the same cases already shown in Fig. 8 are presented. It can be observed that the change in the 


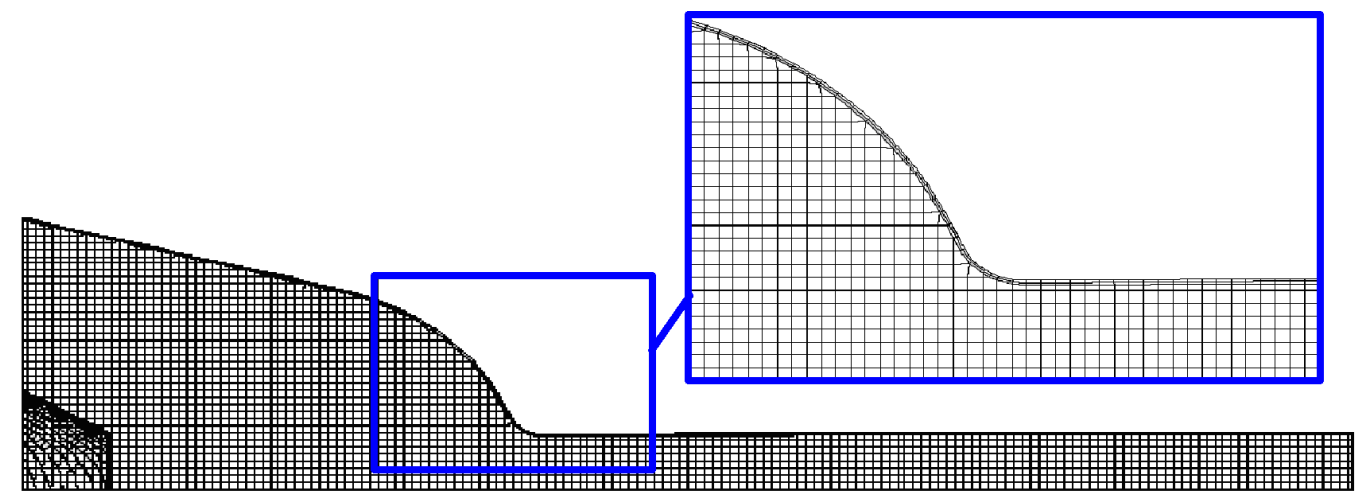

Figure 7: Details of the mesh used for CFD calculations with Star-CD.

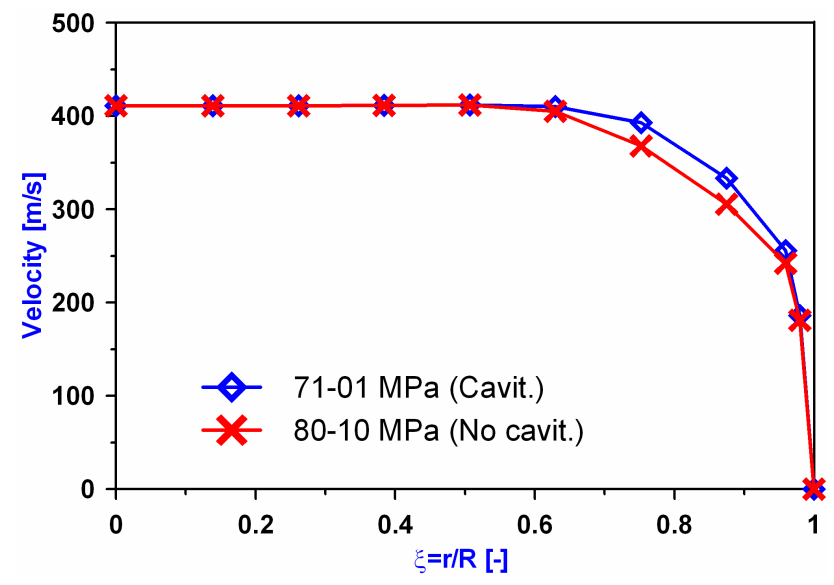

Figure 8: Velocity profiles as a function of the normalized radial coordinate for a cavitating $(71-1 M P a)$ and a non-cavitating $(80-10 M P a)$ case, both simulated with Star-CD using a cavitation model based on the Rayleigh equation. 


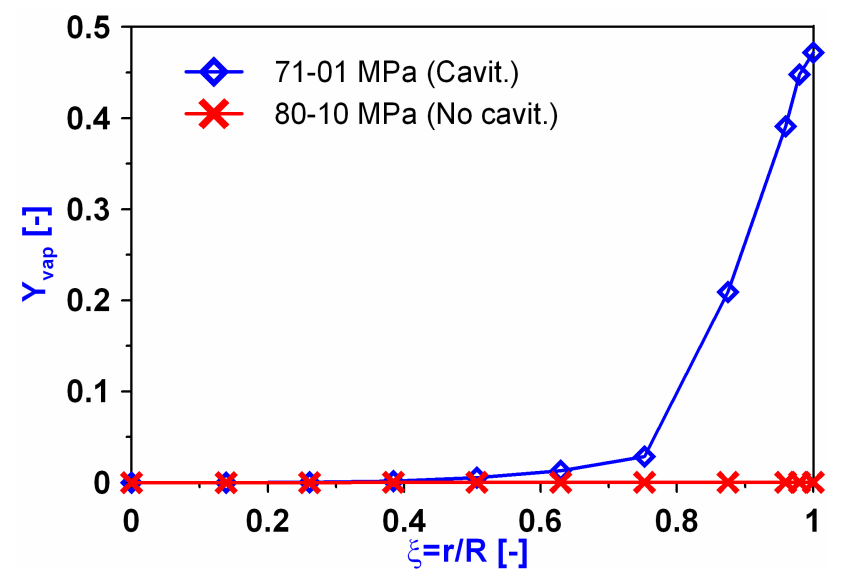

Figure 9: Evolution of the vapor mass fraction as a function of the normalized radial coordinate for the cases shown in Fig. 8.

velocity profile, which is comparable to the one observed when changing the viscosity in the whole section, can be explained by the viscosity variation caused by cavitation, which only takes place near the wall.

To conclude the analysis, the CFD results (for all the simulated cases mentioned at the begining of this section) will be compared with the experimental data already shown in Fig. 2. The comparison will be performed as follows: the increase in velocity (characterized by the $C_{v}$ coefficient) will be plotted as a function of a parameter related to the cavitation intensity (which is the cause for this increase in velocity), characterized e.g. by the $C_{d}$ coefficient. To make the results more intuitive, both parameters are divided by the corresponding value for the non-cavitating case. The same information previously shown in Fig. 2 is now presented in this new way in Fig. 11. In this Figure, the same information corresponding to the CFD simulations are also shown. It can be observed that the simulations agree with the ex- 


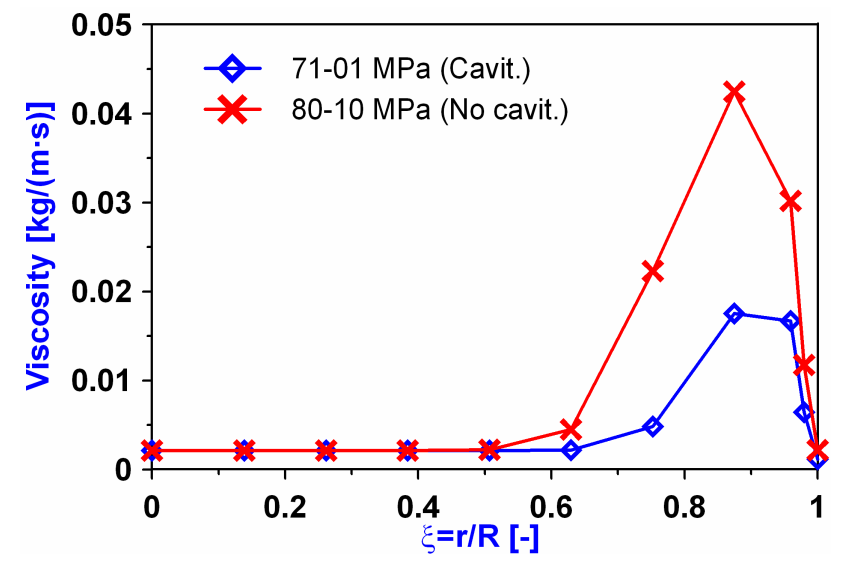

Figure 10: Evolution of the effective viscosity as a function of the normalized radial coordinate for the cases shown in Fig. 8.

periments, thus still giving some more support to the results of the analysis presented in this paper.

\section{Conclusions}

The main conclusions at the end of this study are the following:

- Cavitation has a direct effect on the fuel mass flow rate, which is significantly reduced because of the choke produced by cavitation.

- Experimentally it is observed that the momentum flux is also reduced due to cavitation, but to a lesser extent compared to the mass flow rate. This fact, together with the previous one, leads to an increase in effective injection velocity.

- The theoretical development applied to the simplified case studied here shows that cavitation does not have a direct effect on the effective 


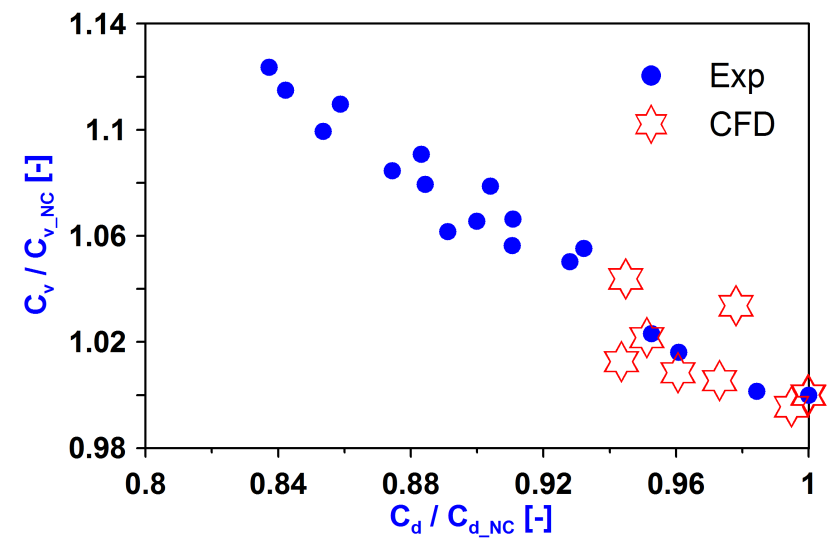

Figure 11: $C_{v}$ versus $C_{d}$ variations for the experimental cases shown in Fig. 2. The same information for the CFD results is also shown.

injection velocity (because, in principle, it wouldn't be modified). But as a two-phase flow exists, the behavior is much more complex. In fact, as some vapor appears near the orifice walls, the fluid viscosity reduces, thus diminishing friction. This has a consequence that can be looked at from two complementary points of view: (1) there are less losses of momentum flux in this region of the nozzle, and (2) the velocity profile is modified, changing to a more "top hat" shape. This change in the velocity profile can harmonize an important reduction in mass flow rate with a less important decrease in momentum flux, in such a way that the effective injection velocity, i.e. the ratio of these two magnitudes, increases (in agreement with the experimental observations). 


\section{Acknowledgements}

The authors would like to thank different members of the CMT-Motores Térmicos team of the Universitat Politècnica de València for their contribution to this work: to Jaime Gimeno, for his fruitful comments, and to Xandra Margot, Stavroula Patouna and Gabriela Bracho for their help in the CFD calculations. They also thank the Ministerio de Educación of Spain for granting the PhD. studies of Oscar A. de la Garza (FPU program), as well as for supporting the VeLoSoot project (TRA2008-06448), as most of this research was performed under the frame of this project.

OpenFOAM and Star-CD are registered trademarks of OpenCFD Ltd. and CD-Adapco, respectively. 


\section{Notation}

$\begin{array}{ll}A & \text { Cross-sectional area } \\ C_{d} & \text { Discharge coefficient } \\ C F D & \text { Computational Fluid Dynamics } \\ C_{M} & \text { Momentum flux coefficient } \\ C_{v} & \text { Velocity coefficient } \\ F & \text { Force } \\ H C C I & \text { Homogeneous Charge Compression Ignition } \\ K & \text { Nurick Cavitation Number }\left(\frac{p_{i n j}-p_{v a p}}{p_{i n j}-p_{b a c k}}\right) \\ L T C & \text { Low Temperature Combustion } \\ \dot{m} & \text { Mass flow rate } \\ \dot{M} & \text { Momentum flux } \\ p & \text { Pressure } \\ P C C I & \text { Premixed Charge Compression Ignition } \\ r & \text { Radial coordinate } \\ R & \text { Radius } \\ R e & \text { Reynolds number } \\ R N G & \text { ReNormalization Group } \\ u & \text { Velocity } \\ y+ & \text { Dimensionless wall distance } \\ Y & \text { Mass fraction } \\ & \end{array}$

Greek symbols 


$\begin{array}{ll}\Delta p & \text { Pressure drop } \\ \mu & \text { Dynamic viscosity } \\ \xi & \text { Normalized radial coordinate }(r / R)\end{array}$

\section{Subscripts}

$\begin{array}{ll}1,2 & \text { In sections } 1,2 \\ \text { back } & \text { In the enclosure where the fuel is injected } \\ \text { berno } & \text { Referred to Bernoulli (theoretical) } \\ c & \text { In section } c \text { (minimum cross-sectional area) } \\ \text { eff } & \text { Effective } \\ \text { inj } & \text { Referred to injection } \\ L & \text { Referred to liquid } \\ m & \text { Referred to the mixture } \\ N C & \text { No Cavitation } \\ \text { o } & \text { At the orifice exit } \\ \text { vap } & \text { Referred to vapor } \\ w \_c a v & \text { With cavitation } \\ w / o \_c a v & \text { Without cavitation } \\ \text { wall } & \text { Referred to the wall } \\ x & \text { In the axial direction }\end{array}$




\section{References}

[1] Gan S., Ng H.K. y Pang K.M. "Homogeneous Charge Compression Ignition (HCCI) combustion: implementation and effects on pollutants in direct injection diesel engines". Applied Energy, Vol. 88, pp. 559-567, 2011.

[2] Torregrosa A.J., Broatch A., Novella R. y Mónico L.F. "Suitability analysis of advanced diesel combustion concepts for emissions and noise control". Energy, Vol. 36, pp. 825-838, 2011.

[3] Thurnheer T., Edenhauser D., Soltic P., Schreiber D., Kirchen P. y Sankowski A. "Experimental investigation on different injection strategies in a heavy-duty diesel engine: emissions and loss analysis". Energy Conversion and Management, Vol. 52, pp. 457-467, 2011.

[4] Arrègle J., Pastor J.V., López J.J. y García A. "Insights on postinjection-associated soot emissions in direct injection diesel engines". Combustion and Flame, Vol. 154, pp. 448-461, 2008.

[5] Suh H.K., Yoon S.H. y Lee C.S. "Effect of multiple injection strategies on the spray atomization and reduction of exhaust emissions in a compression ignition engine fueled with dimethyl ether (DME)". Energy Fuels, Vol. 24, pp. 1323-1332, 2010.

[6] Ozsezen A.N. y Canakci M. "Determination of performance and combustion characteristics of a diesel engine fueled with canola and waste palm oil methyl esters". Energy Conversion and Management, Vol. 52, pp. 108-116, 2011. 
[7] Qi D.H., Chen H., Geng L.M. y Brian Y.ZH. "Experimental studies on the combustion characteristics and performance of a direct injection engine fuelled with biodiesel/diesel blends". Energy Conversion and Management, Vol. 51, pp. 2985-2992, 2010.

[8] Karra P.K. y Kong S.C. "Experimental study on effects of nozzle hole geometry on achieving low diesel engine emissions". J. Eng. Gas Turbine Power, Vol. 132, pp. 022802-022811, 2010.

[9] Som S., Aggarwal S.K., El-Hannouny E.M. y Longman D.E. "Investigation of nozzle flow and cavitation characteristics in a diesel injector". Journal of Engineering for Gas Turbines and Power, Vol. 132, pp. 2802$2814,2010$.

[10] Yi Y. y Reitz R.D. "Modeling the effect of primary atomization on diesel engine emissions". SAE Transactions, Section 3, Journal of Engines (SAE Paper 2003-01-1041), Vol. 112, pp. 1311-1330, 2003.

[11] Som S. y Aggarwal S.K. "Effects of primary breakup modeling on spray and combustion characteristics of compression ignition engines". Combustion and Flame, Vol. 157, pp. 1087-1096, 2010.

[12] Kampmann S., Dittus B., Mattes P. y Kirner M. "The influence of hydro grinding at VCO nozzles on the mixture preparation in a DI diesel engine". SAE Paper 960867, 1996.

[13] Payri R., Salvador F.J., Gimeno J. y Zapata D.L. "Diesel nozzle geometry influence on spray liquid-phase fuel penetration in evaporative conditions". Fuel, Vol. 87, pp. 1165-1176, 2008. 
[14] Kent J.C. y Brown G.M. "Nozzle exit flow characteristics for squareedged and rounded inlet geometries". Combustion Science and Technology, Vol. 30, pp. 121-132, 1983.

[15] Payri R., Molina S., Salvador F.J. y Gimeno J. "A study of the relation between nozzle geometry, internal flow and spray characteristics in diesel fuel injection system". KSME International Journal, Vol. $18 \mathrm{n}^{\circ} 7$, pp. 1222-1235, 2004.

[16] Soteriou C., Andrews R. y Smith M. "Direct injection diesel sprays and the effect of cavitation and hydraulic flip on atomization". SAE Paper 950080, 1995.

[17] Suh H.K. y Lee C.S. "Effect of cavitation in nozzle orifice on the diesel fuel atomization characteristics". International Journal of Heat and Fluid Flow, Vol. 29, pp. 1001-1009, 2008.

[18] Desantes J.M., Payri R., Salvador F.J. y de la Morena J. "Influence of cavitation phenomenon on primary break-up and spray behavior at stationary conditions". Fuel, Vol. 89, pp. 3033-3041, 2010.

[19] Payri R., García J.M., Salvador F.J. y Gimeno J. "Using spray momentum flux measurements to understand the influence of diesel nozzle geometry on spray characteristics". Fuel, Vol. 84, pp. 551-561, 2005.

[20] Payri F., Payri R., Salvador F.J. y Martínez-López J. "A contribution to the understanding of cavitation effects in diesel injector nozzles through a combined experimental and computational investigation". Computers and Fluids, Vol. 58, pp. 88-101, 2012. 
[21] Nurick W.H. "Orifice cavitation and its effect on spray mixing". Journal of Fluids Engineering, Vol. 98, pp. 681-687, 1976.

[22] Desantes J.M., Arrègle J., López J.J. y Hermens S. "Experimental characterization of outlet flow for different diesel nozzle geometries". SAE Paper 2005-01-2120, 2005.

[23] Schmidt D.P. y Corradini M.L. "Analytical prediction of the exit flow of cavitating orifices". Atomization and Sprays, Vol. 7 no 6, pp. 603-616, 1997.

[24] Nakayama Y. y Boucher R.F. Introduction to fluid mechanics. Butterworth/Heinemann, 1999.

[25] OpenFOAM. OpenFOAM. Web site: http://www.openfoam.com. 2010.

[26] Kärrholm F.P. Numerical modelling of diesel spray injection, turbulence interaction and combustion. Tesis Doctoral, Chalmers Institute of Technology, Göteborg (Sweeden), 2008.

[27] Salvador F.J., Romero J.V., Roselló M.D. y Martínez-López J. "Validation of a code for modeling cavitation phenomena in diesel injector nozzles". Mathematical and Computer Modelling, Vol. 52, pp. 11231132, 2010.

[28] Payri R., Tormos B., Gimeno J. y Bracho G. "The potential of large eddy simulation (LES) code for the modeling of flow in diesel injectors". Mathematical and Computer Modelling, Vol. 52, pp. 1151-1160, 2010. 
[29] Versteeg H.K. y Malalasekera W. An introduction to CFD: the finite volume method. Longman, 1995.

[30] Soteriou C., Andrews R. y Smith M. "Further studies of cavitation and atomization in diesel injection". SAE Paper 1999-01-1486, 1999.

[31] Rayleigh Lord. "On the pressure developed in a liquid during the collapse of a spherical cavity". Philosophical Magazine, Vol. 34, pp. 94-98, 1917.

[32] Payri F., Margot X., Patouna S., Ravet F. y Funk M. "A CFD study of the effect of the needle movement on the cavitation pattern of diesel injectors". SAE Paper 2009-24-0025, 2009.

[33] Margot X., Hoyas S., Gil A. y Patouna S. "Numerical modelling of cavitation: validation and parametric studies". Engineering Applications of Computational Fluid Mechanics, Vol. 6, pp. 15-24, 2012.

[34] Margot X., Hoyas S., Fajardo P. y Patouna S. "CFD study of needle motion influence on the exit flow conditions of single-hole injectors". Atomization and sprays, Vol. 21, pp. 31-40, 2011. 